\title{
Postural and Muscular Activity Changes in Camptocormia Patients as a Result of Rehabilitation Treatment with the TPAD Brace
}

\author{
Ricardo Duarte ${ }^{1 *}$, Michel Mesnard ${ }^{1}$, Etienne Guillaud ${ }^{2}$ and Mathieu de Sèze ${ }^{3}$ \\ ${ }^{1}$ Université de Bordeaux, Institut de Mécanique et d'Ingénierie, France \\ ${ }^{2}$ Institut de Neurosciences Cognitives et Intégratives d'Aquitaine, France
}

${ }^{3}$ Centre Hospitalier Universitaire de Bordeaux, France

*Corresponding author: Ricardo Duarte, Université de Bordeaux, Institut de Mécanique et d'Ingénierie, France.

Received Date: November 12, 2018

Published Date: November 29, 2018

\begin{abstract}
Camptocormia is a postural disease that affects the elderly population and is characterized by the anteflexion of the trunk. Orthopedic medical devices, such as the Thoracic Pelvic Anterior Distractor brace, are used in combination with physiotherapy during the treatment.

It is therefore important to evaluate the changes that this brace can make to posture and the erector spinae muscle performance. During this study, a postural evaluation was performed in twenty camptocormia patients by quantifying the sagittal arrows, with and without the brace, during walking. The erector spinae muscular intensity was evaluated by measuring muscular activity at the C7, T12, L3 and S1 vertebral processes during static and dynamic exercises.

Throughout this study, it was possible to observe the influence of the TPAD brace in reestablishing the natural upright posture by reducing significantly the C7-SAR (sagittal arrow) and Patella-SAR, which decreased from $21 \mathrm{~cm}$ to $9 \mathrm{~cm}$ and from $27 \mathrm{~cm}$ to $17 \mathrm{~cm}$ respectively.

It was observed that muscular intensity decreased significantly in all evaluated vertebral levels when patients used the brace in static positions. The non-significant change in muscular activity during walking (from beginning to end) suggests that the brace promotes stable muscular activity in the erector spinae muscles.
\end{abstract}

Keywords: Vertebral alignment; Electromyography; Walking gait; Posture; Brace; Movement analysis

\section{Introduction}

Camptocormia, also known as "bent spine syndrome", produces a progressive anteflexion of the trunk in the sagittal plane when sitting, standing and walking [1-8]. One particular feature of camptocormia is that it is reduced when patients are in a supine position or when they can actively straighten up by pushing with the hands on the pelvis or with the back against the wall $[1,9,10]$.

Camptocormia can have considerable impacts: lumbar pain, problems with breathing, balance and social interactivity interference $[2,10]$. In addition, the flexion of the trunk results in a displacement of the body's center of gravity towards the front, which the patient compensates for by bending the knees (Figures 1A-1C).

Although camptocormia has not been studied a great deal, it can be said that the initial signs appear in 65-75-year-olds, and that among these, women are more affected than men $75 \%$ of cases) [2,5,11-14]. However, little is known about its origins and it poses a challenge to the medical community because of the wide range of possible etiologies. 15,16 According to current knowledge, there are two accepted theories on the origins of the condition: myopathy (associated with Parkinson's disease) and/or dystonia 
of the paravertebral muscles (aberrant contraction of the muscles that bend the trunk) $[2,14,17-21]$.

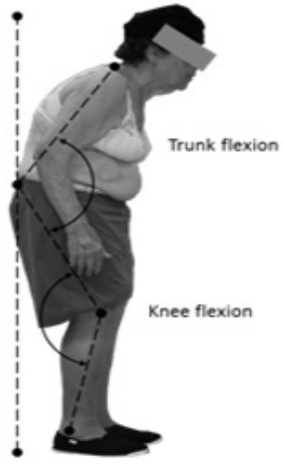

(A)

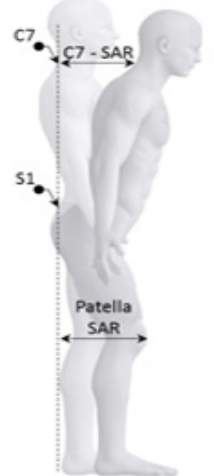

(B)

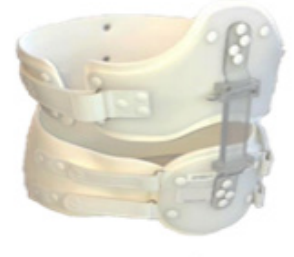

Camptocormia brace

(C)

Figure 1: A: Typical posture B: Postural arrows C: TPAD brace developed by Lagarrigue S.A.

Although current knowledge does not provide sufficient evidence as to its origin, two types of camptocormia can be identified according to the degree of correction possible: totally reducible (the vertebral column remains partially flexible and malleable) or partly reducible (the vertebral column is not very malleable and full straightening is not possible) $[2,12]$.

The recommended treatment will clearly be influenced by the potential causes. One treatment that is well accepted by the medical community and which gives satisfactory results combines physiotherapy exercises and the use of an external device, a brace [4,22-25]. The aim of the physiotherapy is to strengthen the extensor muscles of the back, responsible for straightening the trunk. At the same time, the function of the brace is to provide postural support during daily activities and, in the long term, to help restore the natural posture of the spine.

Camptocormia patients are currently assessed by carrying out a static analysis using X-rays. To complete this analysis, we propose a static and a dynamic study of changes in posture and muscular activity as a result of using the TPAD brace since it is an oftenprescribed orthosis in the treatment of camptocormia.

\section{Methods}

\section{Sample}

Due to the origins of camptocormia, we selected only patients with idiopathic camptocormia, based on preliminary clinical evaluation.

A sample consisting of twenty idiopathic camptocormia patients was evaluated, all with dominant right leg, aged from 68 to 86 years old $(77 \pm 9)$, weighing between 50 and $118(70 \pm 11)$ kilograms', measuring between 148 and 187 (165 \pm 10$)$ centimeters and with a body mass index (BMI) of $24 \pm 5 \mathrm{~kg} \cdot \mathrm{m}^{-2}$.

\section{Preparing the patients}

The patients were dressed in shorts and sandals. Their skin was cleaned using alcohol to improve adhesion of the measuring equipment to the skin as well as the skin conductance.

When patients were not wearing the brace, twenty reflective markers (Optitrack reflective markers 7/16") were placed on the spinous processes of vertebrae C7, T3, T7, T12, L3, S1 and on each knee and each foot (Figure 2A).

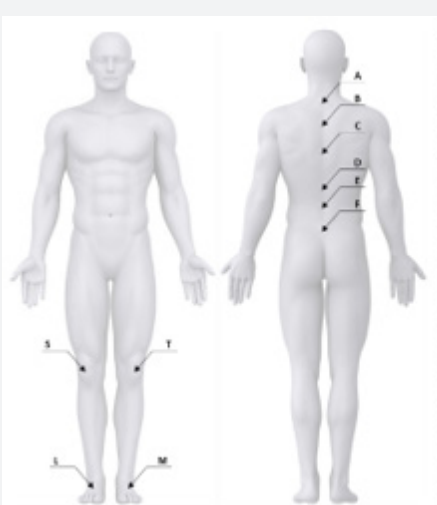

(A)

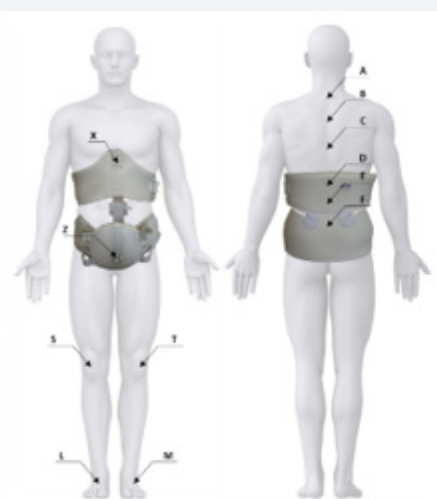

(8)

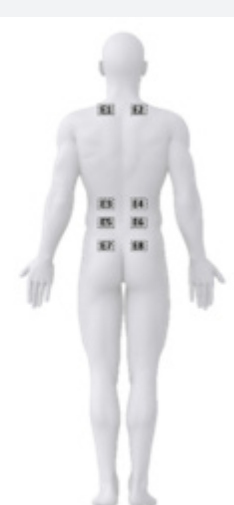

(C)

Figure 2: Placing the devices A: reflective markers on patient without brace B: reflective markers on patient with brace C: surface EMG electrodes. 
When they were wearing the brace, the markers on the spinous processes of vertebrae T12, L3 and S1 were moved onto the outside of the brace but kept at the level of the corresponding vertebral process. Two additional markers were placed in the upper and lower part of the brace's distraction mechanism (Figure 2B). Motion capture was recorded using a frequency of $120 \mathrm{~Hz}$ through Natural Point Flex - 13 cameras $(n=24)$.

To evaluate muscular activity, twelve surface electromyography electrodes (Delsys Trigno $囚$ ) were placed on the patients along the direction of the muscle fibers of the spinal erectors close to the spinous processes of vertebrae C7, T12, L3 and S1, following the protocol defined by De Sèze and Cazalets [26]. When the brace was used, the electrodes placed along the spinal column were retained, as they did not affect the positioning of the brace (Figure $2 \mathrm{C}$ ).

\section{Static tests}

Tests to measure change in the postural arrows and in muscular activity in a static position were carried out on a force platform (ATMI®) in three phases:

a. In the first phase patients were asked to stand in their most usual (spontaneous) position, in order to quantify their camptocormia posture.

b. In the second phase, patients were asked to straighten up in order to evaluate their ability to actively adopt the redressed position.

c. In the third phase, the patient was put into the TPAD brace prescribed by the physician and asked to straighten up into the possible palliative position.
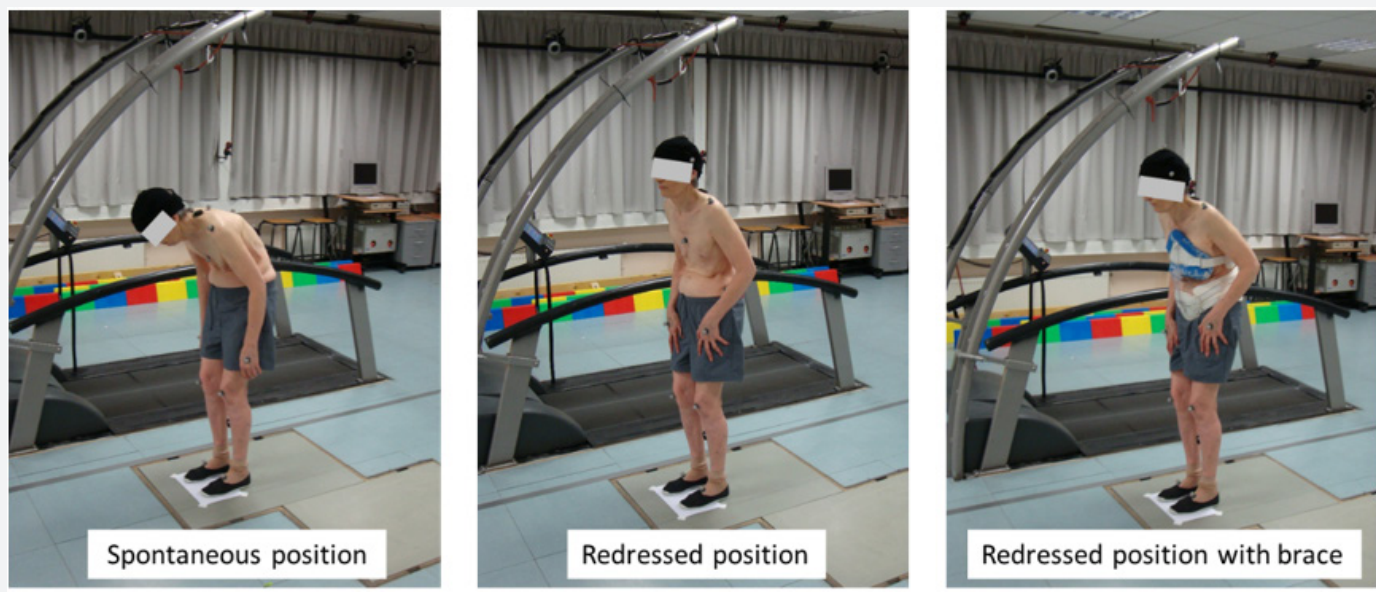

Figure 3: Positions during static tests.

All static tests were of 30 seconds duration in an immobile position (Figure 3).

\section{Walking tests}

Patients were asked to walk around the platform at their usual speed, along a $30 \mathrm{~m}$ rectangular pathway, for a maximum of $10 \mathrm{~min}$, both without and then with the TPAD brace. The recording area was limited to a straight-line area of about $5 \mathrm{~m}$ since it was considered the ideal recording area.

Results for change in posture and muscular activity will be divided into:

a. postural analysis of arrows (C7-SAR, T3-SAR, T7-SAR, T12-SAR, L3-SAR and patella-SAR) in static position,

b. postural analysis of knees,

c. postural analysis of arrows (C7-SAR, T3-SAR, T7-SAR, T12-SAR, L3-SAR and patella-SAR) while walking,

d. analysis of muscular activity in static position,

e. analysis of muscular activity while walking

\section{Data processing}

Kinematic data were processed using Motive Optitrack software. Signal loss compensation was carried out and a $10 \mathrm{~Hz}$ low pass filter (reverse Butterworth) was applied.

Electromyography data were processed using aware toolbox developed by the Movement Analysis Platform (PAM) at the Institute of Cognitive and Integrative Neurosciences of Aquitaine (CNRS Bordeaux). The raw data were filtered with a $30 \mathrm{~Hz}$ highpass filter, rectified and filtered with a $3 \mathrm{~Hz}$ low-pass filter using a fourth-order reverse Butterworth filter. During the static tests, muscular activity was normalized according to each patient's spontaneous upright position. The average of EMG value recorded during this phase became $100 \%$, and the EMG from all other tasks were normalized and expressed in percentage of this static phase.

During walking, the start of each cycle was identified by the change in velocity of the movement of the right foot in the sagittal plane (a new cycle begins when the velocity increase over the threshold of Xm. s-2). In this way, each cycle could be broken down and the EMG data could be normalized in the time domain at the cycle average duration. The average EMG signal and averaged cycle 
area (surface covered by the rectified EMG trace during cycle) were calculated for each patient. Electromyography data were also normalized for each subject based on the start of the walk without the brace.

\section{Results}

\section{Postural analysis of arrows in static positions}

Table 1: Sagittal arrow at different vertebra levels.

\begin{tabular}{|c|c|c|c|c|c|}
\hline & C7-SAR & T3-SAR & T7-SAR & T12-SAR & L3-SAR \\
\hline Spontaneous & $20.96 \pm 8.71$ & $16.10 \pm 4.32$ & $9.24 \pm 2.61$ & $3.37 \pm 1.03$ & $2.43 \pm 0.81$ \\
\hline Redressed & $15.22 \pm 5.06$ & $12.06 \pm 3.20$ & $6.65 \pm 1.47$ & $2.41 \pm 1.09$ & $1.84 \pm 0.55$ \\
\hline Brace & $9.31 \pm 3.17$ & $6.47 \pm 2.62$ & $3.51 \pm 1.01$ & 0.18 & 0.49 \\
\hline *P(spontaneous/redressed) & 0.46 & 0.16 & 0.33 & $\mathrm{P}<0.001$ & 0.14 \\
\hline$*$ P (spontaneous/ brace) & $\mathrm{P}<0.001$ & $\mathrm{P}<0.001$ & $\mathrm{P}<0.001$ & \\
\hline
\end{tabular}

\section{${ }^{*}$ ANOVA}

Static postural analysis of the sagittal arrows gives different results according to the position under consideration (Table 1).

By comparing the spontaneous standing position with the redressed standing position (active straightening of the trunk), we see a non-significant decrease $(p>0.05)$ in the postural arrows at all levels considered.

However, it should be noted that patients were not able to straighten up fully, and none of them brought the C7-SAR arrow to zero, as can be seen in non-camptocormic subjects.

When the brace was used, a significant decrease was observed $(\mathrm{P}<0.05)$ in the postural arrows at levels $\mathrm{C} 7, \mathrm{~T} 3, \mathrm{~T} 7$ and $\mathrm{T} 12$ compared with the spontaneous position without the brace. At level L3, there was no significant decrease in the L3-SAR postural arrow.

\section{Trunk straightening behavior}

Although a decrease in the arrows was observed for active straightening and when the brace was used, it should also be noted that the trunk straightening behavior was not the same for all patients.

Some patients, described as "pivoting", seemed to tilt forward around a single rotation axis; their postural arrows were the result of the rotation between two vertebral processes that were very close together. A second group of patients, called "rolling", seemed to have a continuous collapse of the entire trunk, resulting from an accumulation of rotations at several levels; the relationship between postural arrows as a result of two successive rotations was virtually constant (Table 2).

In order to explore this phenomenon, the change in ratios between C7-SAR, T3-SAR, T7-SAR, T12-SAR and L3-SAR sagittal arrows was studied to characterize trunk flexion behavior in the camptocormia patient.

\section{Test schedule}

All tests were carried out from 9.30 am to reduce the effect of muscle fatigue as a result of daily activities.
Analysis of Table 2 shows a rolling behavior in $60 \%$ of subjects and a pivoting behavior in $40 \%$.

Table 2: Evaluation of pivoting and rolling patients.

\begin{tabular}{|c|c|c|c|c|}
\hline & C7/T3 & T3/T7 & T7/T12 & T12/L3 \\
\hline Pivoting average & 4.63 & 1.2 & 1.22 & 0.99 \\
\hline Rolling average & 0.94 & 1.2 & 1.1 & 1.2 \\
\hline Pivoting Patients & $40 \%$ & \multicolumn{2}{|c|}{ Rolling Patients } & $60 \%$ \\
\hline
\end{tabular}

\section{Postural analysis of the knees in static positions}

Flexion of the knees in the posture of camptocormia patients is a compensatory effect to maintain the center of gravity above the polygon of sustentation, despite the anterior flexion of the spine [27]. Thus, measuring the arrow on the patella (or patellaSAR) in the sagittal plane gave the difference between the three positions under consideration: spontaneous position, redressed position without the brace and with the brace. It was observed for the spontaneous position a patella-SAR of $27.35 \pm 9.35$, for the redressed position $21.53 \pm 7.58$ and using brace $17.53 \pm 5.79$.

Considering an ANOVA statistical test, we observed that the sagittal arrow values decreased in a way that was statistically nonsignificant $(p>0.05)$ when the patient straightened up from the spontaneous position. The decrease was greater and statistically significant $(\mathrm{p}<0.05)$ when comparing the upright position wearing the brace with the spontaneous position without the brace.

\section{Postural analysis of arrows during walking}

Analysis of the arrows focused on measuring changes in sagittal arrows at levels C7, T3, T7, T12 and L3 between the beginning and the end of the walking exercise. The first passage was considered as the beginning of the walking test and the last passage as the end of the walking test (Table 3) 
Table 3: Change in postural arrows during walking.

\begin{tabular}{|c|c|c|c|c|c|}
\hline & C7-SAR & T3-SAR & T7-SAR & T12-SAR & L3-SAR \\
\hline Beginning $w / o$ brace & $21.50 \pm 6.23$ & $15.75 \pm 5.24$ & $10.81 \pm 4.42$ & $6.37 \pm 1.74$ & $3.41 \pm 0.75$ \\
\hline Beginning w/ brace & $17.70 \pm 5.57$ & $11.63 \pm 4.68$ & $7.71 \pm 2.99$ & $3.80 \pm 1.27$ & $1.67 \pm 0.56$ \\
\hline End w/o brace & $26.53 \pm 6.81$ & $19.63 \pm 6.19$ & $13.63 \pm 5.14$ & $8.26 \pm 1.83$ & $4.98 \pm 0.91$ \\
\hline End w/ brace & $19.46 \pm 5.89$ & $12.88 \pm 5.05$ & $8.70 \pm 3.28$ & $4.88 \pm 1.147$ & $2.06 \pm 0.75$ \\
\hline \multicolumn{6}{|c|}{ Ratios (\%) } \\
\hline (D-F)/D (w/o brace) & $23 \%$ & $25 \%$ & $26 \%$ & $30 \%$ & $85 \%$ \\
\hline (D-F)/D (w/ brace) & $10 \%$ & $11 \%$ & $13 \%$ & $28 \%$ & $23 \%$ \\
\hline (S-A)/S (beginning) & $18 \%$ & $26 \%$ & $29 \%$ & $40 \%$ & $95 \%$ \\
\hline (S-A)/S (end) & $27 \%$ & $34 \%$ & $36 \%$ & $41 \%$ & $59 \%$ \\
\hline \multicolumn{6}{|c|}{ Key: D - Beginning / F - End / S - w/o brace / A - w/ brace } \\
\hline \multicolumn{6}{|c|}{ Statistical comparison: beginning without brace - beginning with brace } \\
\hline *p & 0.36 & 0.352 & 0.096 & 0.019 & 0.034 \\
\hline \multicolumn{6}{|c|}{ Statistical comparison: end without brace - end with brace } \\
\hline *p & 0.278 & 0.292 & 0.079 & 0.001 & 0.04 \\
\hline \multicolumn{6}{|c|}{ Statistical comparison: beginning without brace - end without brace } \\
\hline *p & 0.332 & 0.317 & 0.291 & 0.374 & 0.015 \\
\hline \multicolumn{6}{|c|}{ Statistical comparison: beginning with brace - end with brace } \\
\hline *p & 0.421 & 0.379 & 0.329 & 0.123 & 0.132 \\
\hline \multicolumn{6}{|c|}{ Statistical comparison: ratio (D-F/D) without - (D-F/D) with } \\
\hline *p & 0.045 & 0.037 & 0.006 & 0.002 & 0.038 \\
\hline
\end{tabular}

*ANOVA

We observed a tendency for the values of the postural arrows to increase during walking at all vertebral levels. Increases in the upper levels (C7, T3 and T7) were more pronounced when patients were not wearing the brace.

When comparing the beginning without the brace and the beginning with the brace, no significant difference was observed ( $p>0.05$ ) at vertebral levels C7, T3 and T7. However, values for arrows at vertebral levels T12 and L3 were significantly higher when the patient was not wearing the brace.

When comparing the end without the brace and the end with the brace, a systematic though not significant decrease was observed ( $p>0.05$ ) in arrows C7, T3 and T7 when patients wore the brace. In addition, a significant decrease $(\mathrm{p}<0.05)$ in arrows T12 and L3 was observed when patients wore the brace.

When comparing the beginning and the end of walking without the brace a statistically significant accentuation in the L3 arrow $(p<0.05)$ was observed. However, increases in the arrows were not statistically significant $(p>0.05)$ at levels $\mathrm{C} 7, \mathrm{~T} 3, \mathrm{~T} 7$ and T12.

No significant difference was observed when comparing sagittal arrows at the beginning and end of the walking test with the brace.

In order to explore the worsening of the arrows during the walking test with and without the brace, the percentages of individual worsening of the initial and final arrows with and without the brace were quantified using the following expressions: (début-fin)/fin without and with a brace. Significant differences $(p<0.05)$ were observed at all levels.

\section{Analysis of muscular activity under static conditions in different positions}

The characteristics of muscular activity in a camptocormic subject are not the same as the muscular activity of a healthy individual. In healthy individuals it is very easy to distinguish the typical exhalation associated with muscular activation during effort (e.g. when walking), but in the case of camptocormic subjects this exhalation is much less evident.

Muscular activity was compared in three different situations in order to evaluate the effects of the brace. For the trunk, it was evaluated in the spontaneous and the redressed position without the brace and in the redressed position with the brace.

It was observed that muscular activity in the back in the redressed position was significantly greater $(p<0.05)$ at all levels than muscular activity in the back in the spontaneous position. This increase was particularly evident for vertebra C7 (Table 4).

When the brace was used, however, we observed a significant decrease $(\mathrm{p}<0.05)$ in this muscular activity at all levels compared with activity in the spontaneous position. This decrease was particularly evident around the brace, especially at levels T12, L3 and S1 (Table 4).

In addition, a significant decrease $(\mathrm{P}<0.05)$ could be seen between the redressed position and the redressed position with 
the brace (Table 4). It was also clear that there were no significant observation was to be expected, since only patients with no lateral differences $(\mathrm{P}>0.05)$ between the right and left sides. This deviation of the spine were selected.

Table 4: Ratio of muscular activity for three situations.

\begin{tabular}{|c|c|c|c|c|c|c|c|c|}
\hline & \multicolumn{2}{|c|}{ C7 } & \multicolumn{2}{|c|}{ T12 } & \multicolumn{2}{|c|}{ L3 } & \multicolumn{2}{|c|}{ S1 } \\
\hline & Left & Right & Left & Right & Left & Right & Left & Right \\
\hline Spont./Redres. & $0.76 \pm 0.25$ & $0.79 \pm 0.24$ & $0.80 \pm 0.29$ & $0.94 \pm 0.26$ & $0.89 \pm 0.28$ & $0.93 \pm 0.32$ & $0.88 \pm 0.28$ & $0.87 \pm 0.21$ \\
\hline$* \mathbf{p}$ & 0.000 & 0.000 & 0.000 & 0.006 & 0.018 & 0.008 & 0.038 & 0.001 \\
\hline Spont./Brace & $1.28 \pm 0.28$ & $1.14 \pm 0.41$ & $1.37 \pm 0.52$ & $1.36 \pm 0.47$ & $1.57 \pm 0.53$ & $1.51 \pm 0.40$ & $1.66 \pm 0.78$ & $1.83 \pm 0.88$ \\
\hline$* \mathbf{p}$ & 0.014 & 0.016 & 0.005 & 0.019 & 0.000 & 0.006 & 0.001 & 0.001 \\
\hline Redres./Brace & $1.82 \pm 0.33$ & $1.80 \pm 0.51$ & $1.41 \pm 0.29$ & $1.75 \pm 0.72$ & $1.77 \pm 0.56$ & $1.44 \pm 0.72$ & $1.41 \pm 0.41$ & $1.56 \pm 0.28$ \\
\hline$* \mathbf{p}$ & 0.021 & 0.000 & 0.012 & 0.001 & 0.000 & 0.000 & 0.002 & 0.000 \\
\hline Left/Right & \multicolumn{3}{|c|}{ Spontaneous position } & \multicolumn{3}{|c|}{ Redressed position } & \multicolumn{2}{|c|}{ Redressed position with brace } \\
\hline$* \mathbf{p}$ & \multicolumn{3}{|c|}{0.22} & \multicolumn{3}{|c|}{0.33} & \multicolumn{2}{|c|}{0.41} \\
\hline
\end{tabular}

*ANOVA

\section{Analysis of muscular activity during walking}

Muscular activity with and without the brace was evaluated at the beginning and the end of the walking test, considering only the first and last laps. Unlike the static upright position at the beginning where we hope to obtain muscular activity close to zero (in the case of a healthy subject), when walking, the body is in a constant state of imbalance and as a result, muscular activity in the upright position will never be zero.

Decreases in the intensity of muscular activity could be seen along all of the spine between the beginning and the end of the walking test without the brace. These decreases were statistically significant at all levels $(\mathrm{p}<0.05)$ except $\mathrm{C}$.
However, when the brace was used, the erector spinae muscles activity intensity showed no significant decrease ( $p>0.05$ ) between the beginning and the end of the walking test.

Comparisons of electromyographic activity at the beginning of the walking test, with and without the brace, revealed a significant increase $(\mathrm{p}<0.05)$ in muscular activity at levels C7 and T12 (Table $5)$ as a result of wearing the brace.

However, a significant decrease $(\mathrm{p}<0.05)$ in muscular activity was observed at levels L3 and S1 and this highlights the predominantly supportive role of the brace at the lumbar levels (Table 5).

Table 5: Ratios for normalized muscular activities along the erector spinae muscles.

\begin{tabular}{|c|c|c|c|c|c|c|c|c|}
\hline & \multicolumn{2}{|c|}{ C7 } & \multicolumn{2}{|c|}{ T12 } & \multicolumn{2}{|c|}{ L3 } & \multicolumn{2}{|c|}{ S1 } \\
\hline & Left & Right & Left & Right & Left & Right & Left & Right \\
\hline w/o brace Beg./End & $1.15 \pm 0.29$ & $1.13 \pm 0.47$ & $1.35 \pm 0.64$ & $1.30 \pm 0.58$ & $1.26 \pm 0.44$ & $1.38 \pm 0.84$ & $1.11 \pm 0.15$ & $1.19 \pm 0.26$ \\
\hline$* \mathbf{p}$ & 0.361 & 0.238 & 0.023 & 0.037 & 0.015 & 0.024 & 0.035 & 0.018 \\
\hline w/ brace Beg./End & $1.07 \pm 0.35$ & $1.05 \pm 0.41$ & $1.10 \pm 0.71$ & $1.04 \pm 0.61$ & $1.01 \pm 0.36$ & $1.07 \pm 0.47$ & $1.08 \pm 0.42$ & $1.07 \pm 0.61$ \\
\hline${ }^{*} \mathbf{p}$ & 0.274 & 0.288 & 0.091 & 0.295 & 0.3611 & 0.491 & 0.297 & 0.234 \\
\hline w/ brace/ w/o brace - beginning & $1.16 \pm 0.66$ & $1.14 \pm 0.39$ & $1.62 \pm 0.28$ & $1.11 \pm 0.74$ & $0.89 \pm 0.31$ & $0.89 \pm 0.22$ & $0.98 \pm 0.37$ & $0.92 \pm 0.40$ \\
\hline$* \mathbf{p}$ & 0.010 & 0.037 & 0.022 & 0.012 & 0.033 & 0.002 & 0.039 & 0.001 \\
\hline w/ /w/o brace - end & $1.30 \pm 0.48$ & $1.29 \pm 0.24$ & $1.25 \pm 0.35$ & $1.13 \pm 0.94$ & $1.08 \pm 0.34$ & $1.09 \pm 0.48$ & $1.03 \pm 0.42$ & $1.16 \pm 0.40$ \\
\hline$* \mathbf{p}$ & 0.024 & 0.017 & 0.026 & 0.018 & 0.019 & 0.015 & 0.071 & 0.087 \\
\hline
\end{tabular}

*ANOVA

\section{Discussion}

\section{Interpretation of static arrows}

The results of the static arrow analysis show that the brace straightens up the patients' posture at several levels, but that it does not completely reduce the postural arrows, especially the C7SAR arrow. This may be linked, on the one hand, to the muscular capacity of the patients' trunk and on the other hand to the lack of spinal flexibility in all patients.
Results for the intrinsic anteflexion behavior of the trunk confirm the existence of two types of behavior

of the trunk in camptocormia patients [21] and these should be incorporated into the design process for orthoses to treat camptocormia. In this way the orthosis components could be adapted to patients' specific needs.

For example, in the case of "pivoting" camptocormia patients, we observed that the axis of rotation is to be found between $\mathrm{C} 7$ and 
T3. The TPAD brace provides support up to T12, according to the geometry of the final support; this may mean that for some patients the brace does not counteract the rotation of the column.

In addition, the variations observed in terms of trunk (pivoting or rolling) flexion between the sexes should be considered in more depth in a further study, to see whether or not these differences are due the composition of the sample.

Observation of the variations in flexion of the knees in this study is in agreement with the bibliography: patients with anteflexion of the trunk need to compensate for the displacement of their center of gravity by bending the knees [28].

These variations also suggest that postural compensation by the knees is less marked when the brace is used; the TPAD straightens up the trunk and, as a result, brings the center of gravity closer to the polygon of sustentation.

\section{Interpretations of walking test}

The results for the walking test beginning with and without the brace, suggest on the one hand, that as a result of wearing the brace, there is a reduction in the pelvic anteversion observed at the beginning of the walk [29] and, on the other hand, the existence of spinal compensation located high up at the beginning of the walk without the brace. This result shows that, since the upper ring of the brace stops at the level of vertebra T12, no correction is possible above this level.

Results for ending without the brace and with the brace, suggest that the brace prevents any increase in the thoracic arrows. This may be linked to the geometry of the brace, especially the body surface area in contact with the support.

Results for beginning and ending without the brace may reflect persistent attempts at spinal compensation associated with the anteversion of the pelvis required in order to walk [30].

Results for beginning and ending with the brace suggest the persistence of the support effect provided by the brace during walking.

For walking without the brace, results at all levels show higher percentages of worsening of the arrows, due to a greater collapse of the trunk.

\section{Interpretation of muscular activity}

Electromyographic evaluation highlighted the importance of using the brace at the level where muscular activity in the back is restored. In contrast to the static upright position, where we hope for muscular activity of around zero for a healthy subject, normal activity when walking should be accompanied by very strong double exhalations [26].

Contrary to what can be seen in the healthy subject, the results for muscular activity during static straightening up by camptocormia patients show that maintaining an upright posture makes considerable demands on the erector spinae muscles.

During walking, the non-significant decrease between the beginning and the end of the test with the brace suggests that this brace contributes to achieving a more stable muscular activity.

Next, we note that using the brace in the static redressed position means that the trunk is supported and reduces the muscular activity of the erectors.

The recordings of muscular activity by the erector spinae in camptocormia subjects reveal radically different muscle behavior from that observed in healthy subjects. We noted continuous activity, whereas De Sèze et al [26] reported virtually no activity in the standing position and phasic activity in bursts during walking.

In addition to these differences, and contrary to muscular activity in static positions, wearing the brace during walking leads to an increase in the muscular activity of the erector spinae, suggesting that the brace promotes muscle expression at the thoracic and cervical levels.

We believe that the decrease in muscular activity observed when walking without the brace is related to a fatigue phenomenon. Thus, maintaining the intensity of muscular activity when walking would appear to be an expression of reduced fatigue. The ultimate aim would be to be able to restore large phasic exhalations, as observed in the healthy subject.

By using the brace, the lumbar pains often noted in camptocormia patients can be reduced [31-33]. Results suggest that the pain is due to excessive and inconsequential muscular activity, of which the only result is that the erector muscles of the back tire more quickly without necessarily producing an effective straightening up.

In this article we present an evaluation of the support provided by the TPAD brace for camptocormia patients both in terms of posture and muscular activity. The biomechanical assessment of the patient, both with and without the brace, shows the contribution the brace makes to reducing postural arrows in static positions and during walking. It has been observed that the brace allows for better postural stability in the redressed position and reduces muscular activity at the lumbar level in immobile positions and also during walking. In addition, comparing results with and without the brace at the beginning of the walking test suggest first of all that the support that the brace provides at the cervical level above vertebra T12 is low, and second that there is a need to produce a greater muscular effort.

\section{Acknowledgments}

The authors acknowledge the Association Nationale de la Recherche et de la Technologie, Lagarrigue Aquitaine S.A.S. and the Institut de Neurosciences Cognitives et Intégratives d'Aquitaine (CNRS UMR5287). 


\section{Conflict of Interest}

No conflict of interest.

\section{References}

1. Delgado A (2009) Camptocormia: discovered again. Kinésithérapie la Revue 9(96): 32-35.

2. Laroche M (2011) La camptocormie du sujet âgé. Rev du Rhum Monogr 78(1): $22-25$

3. Gagliano FS, Ferracane G, Gagliano R (2006) Camptocormia: attività motoria e ortesi. Camptocormia: kinésithérapie et orthèse. Résonances Eur du Rachis 14(44): 1849-1851.

4. Pardessus V, Compere S, Tiffreau V, Blanchard A, Thevenon A (2005) Leather orthesis for treatment of camptocormia: 31 cases. Ann Readapt Med Phys 48(8): 603-609.

5. Jiménez González MM, Pons Serra M, Castano Moreno C, Monés Jiménez L, Martínez Rodenas F (2002) Camptocormia: una enfermedad muscular infrecuente. An Med Interna 19(9): 470-472.

6. Rosen JC, Frymoyer JW (1985) A review of camptocormia and an unusual case in the female. Spine 10(4): 325-327.

7. Soreff J (1983) Camptocormia. Arch Orthop Trauma Surg 101(2): 151152.

8. Lenoir T, Guedj N, Boulu P, Guigui P, Benoist M (2010) Camptocormia: the bent spine syndrome, an update. Eur spine J 19(8): 1229-1237.

9. Laroche M, Cintas P (2010) Bent spine syndrome (camptocormia): a retrospective study of 63 patients. Joint Bone Spine 77(6): 593-596.

10. Tejera B, Holgado S, Calderón V, Pérez R (2013) Camptocormia: case presentation. Reumatol Clínica 9(3): 195-196.

11. Laroche M, Cintas P (2010) Camptocormies ou cormoptoses réductibles: étude rétrospective sur une série de 63 malades. Revue du Rhumatisme $77(6): 621-624$.

12. Bloch F, Houeto JL, Tezenas du Montcel S, Bonneville F, Etchepare F, et al. (2006) Parkinson's disease with camptocormia. J Neurol Neurosurg Psychiatry 77(11): 1223-1228.

13. Bouzgarou E, Dupeyron A, Castelnovo G, Boudousq V, Collombier L, et al. (2007) Camptocormia disclosing Parkinson's disease. Ann Readapt Med Phys 50(1): 55-59.

14. Delcey V, Hachulla E, Michon-Pasturel U, Queyrel V, Hatron PY, et al. (2002) Camptocormia revealing axial myopathy. Concerning seven observations. La Revue de Médecine Interne 23: 144-154.

15. Serratrice G, Pouget J, Pellissier J (1996) Bent spine syndrome. J Neurol Neurosurg Psychiatry 60(1): 51-54.

16. Laroche M, Delisle MB, Aziza R, Lagarrigue J, Mazieres B (1995) Is camptocormia a primary muscular disease? Spine (Phila Pa 1976) 20(9): 1011-1016.
17. Laroche M, Delisle MB (1994) La camptocormie primitive est une myopathie para-vertébrale. Rev Rhum 61(7-8): 481-484.

18. Jankovic J (2010) Camptocormia head drop and other bent spine syndrome heterogeneous etiology and pathogenesis of Parkinson deformities. Mov Disord 25(5): 527-528.

19. Peeraully T, Tan EK (2012) Camptocormia in Parkinson's disease: dystonia or myopathy? Basal Ganglia 2(1): 1-3.

20. Margraf NG, Wrede A, Rohr A, Schulz-Schaeffer WJ, Raethjen J, et al. (2010) Camptocormia in idiopathic Parkinson's disease: a focal myopathy of the paravertebral muscles. Mov Disord 25(5): 542-551.

21. Azher SN, Jankovic J (2005) Camptocormia: pathogenesis, classification, and response to therapy. Neurology 65(3): 355-359.

22. de Sèze MP, Creuzé A, de Sèze M, Mazaux JM (2008) An orthosis and physiotherapy programme for camptocormia: A prospective study. J Rehabil Med 40(9): 761-765.

23. Duarte R, Mesnard M, Wentzy P, Sèze M De (2016) Brace kinematical analysis on patients with camptocormia. Annals of Physical and Rehabilitation Medicine 59: e26.

24. De Sèze M, Creuzé A (2009) Background summary: a new brace for the treatment of camptocormia. Scoliosis 4(Suppl 1): 070.

25. Cugy E, Zauderer J, Dublanc S, De Seze M (2013) Impact d'un corset type DTPA utilisé dans la camptocormie sur les paramètres respiratoires: à propos d'un cas. Annals of Physical and Rehabilitation Medicine 56: e328-e329.

26. Panchoa M, Cazalets DS (2008) Anatomical optimization of skin electrode placement to record electromyographic activity of erector spinae muscles. Surg Radiol Anat 30:137-143.

27. El Helou A (2011) Caractérisation Biomécanique de la cheville et analyse de l'équilibre du sujet agê en reprise d'autonomie 1: 1-192

28. Tramonti C, Di Martino S, Unti E, Frosini D, Bonuccelli U, et al. (2017) Gait dynamics in Pisa syndrome and Camptocormia: The role of stride length and hip kinematics. Gait Posture 57: 130-135.

29. De Seze M, Slugacz L, Guillaud E, Cazalets JR (2010) Étude des moyens de mesure de la camptocormie 96: 86-87.

30. Duval-Beaupère G, Legaye J (2004) Composante sagittale de la statique rachidienne. Revue du Rhumatisme 71(2): 105-119.

31. Sung PS, Lammers AR, Danial P (2009) Different parts of erector spinae muscle fatigability in subjects with and without low back pain. Spine J 9(2): 115-120.

32. Kramer M, Ebert V, Kinzl L, Dehner C, Elbel M, et al. (2005) Surface electromyography of the paravertebral muscles in patients with chronic low back pain. Arch Phys Med Rehabil 86(1): 31-36.

33. Melamed E, Djaldetti R (2006) Camptocormia in Parkinson's disease. J Neurol 253 Suppl 7: VII14- VII16. 